\title{
Correction to: Visualization of microaneurysms using optical coherence tomography angiography: comparison of OCTA en face, OCT B-scan, OCT en face, FA, and IA images
}

\author{
Masafumi Hamada ${ }^{1,2} \cdot$ Kishiko Ohkoshi $^{2} \cdot$ Keiji Inagaki $^{1,2} \cdot$ Nobuyuki Ebihara $^{1,3} \cdot$ Akira Murakami $^{1}$
}

Published online: 24 February 2018

(C) Japanese Ophthalmological Society 2018

Correction to: Japanese Journal of Ophthalmology https://doi.org/10.1007/s10384-018-0570-0

In the original publication, the yellow circles in Figs. $2 a, 2 b$, $3 \mathrm{a}$ and $3 \mathrm{~b}$ are placed incorrectly. The corrected Figs. 2a, $\mathrm{b}$ and $3 a, b$ are given in this Erratum.

The original article was corrected.

The original article can be found online at https://doi.org/10.1007/ s10384-018-0570-0.

Masafumi Hamada

hamamasa@luke.ac.jp

1 Department of Ophthalmology, Juntendo University Graduate School of Medicine, Tokyo, Japan

2 Department of Ophthalmology, St. Luke's International Hospital, 9-1 Akashi-cho, Chuo-ku, Tokyo 104-8560, Japan

3 Department of Ophthalmology, Juntendo University Urayasu Hospital, Urayasu, Japan 

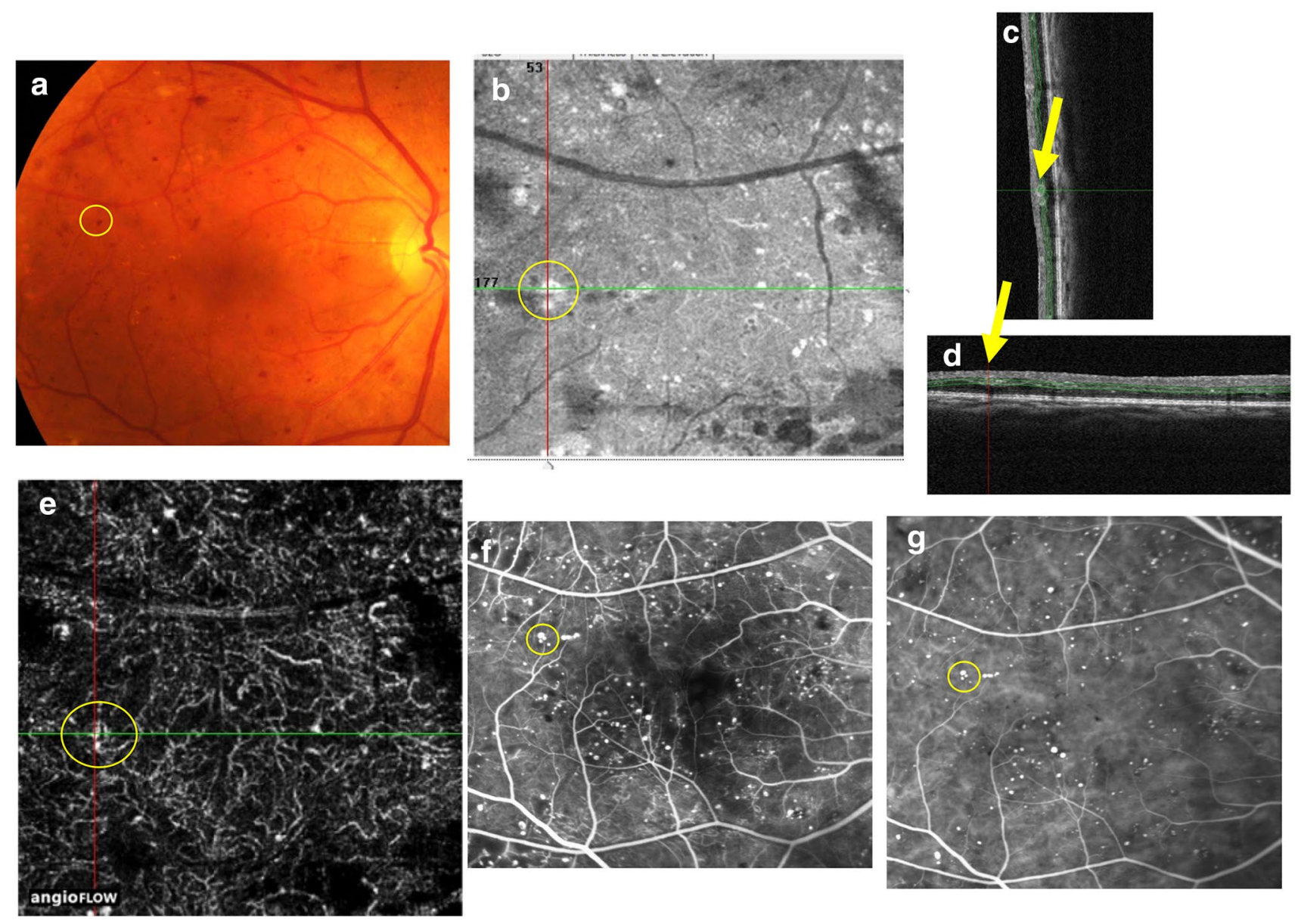

Fig. 2 A 63-year-old man with proliferative diabetic retinopathy of the right eye. a The fundus photograph shows a red punctate spot thought to be a microaneurysm (MA; yellow circle). b The MA is seen in a $3 \times 3$-mm square of an en face image (yellow circle). The MA was confirmed in optical coherence tomography (OCT) B-scan images with the $\mathbf{c}$ vertical and $\mathbf{d}$ horizontal sliders aligned. A ringshape, thought to be the MA, can be seen. The MA lumen was open and cystoid macular edema was observed in the vicinity. e A comma-like finding was observed in a $3 \times 3$-mm square OCT angiography (OCTA) en face image (deep plexus). This was defined as a comma-like-type MA. $\mathbf{f}$ The yellow circle shows hyperfluorescence in a fluorescence angiographic (FA) image (1 minute and 6 seconds) matching the OCTA en face image. $\mathbf{g}$ The yellow circle shows hyperfluorescence in an indocyanine green angiographic (IA) image (2 minutes and 41 seconds) matching the OCTA en face image 

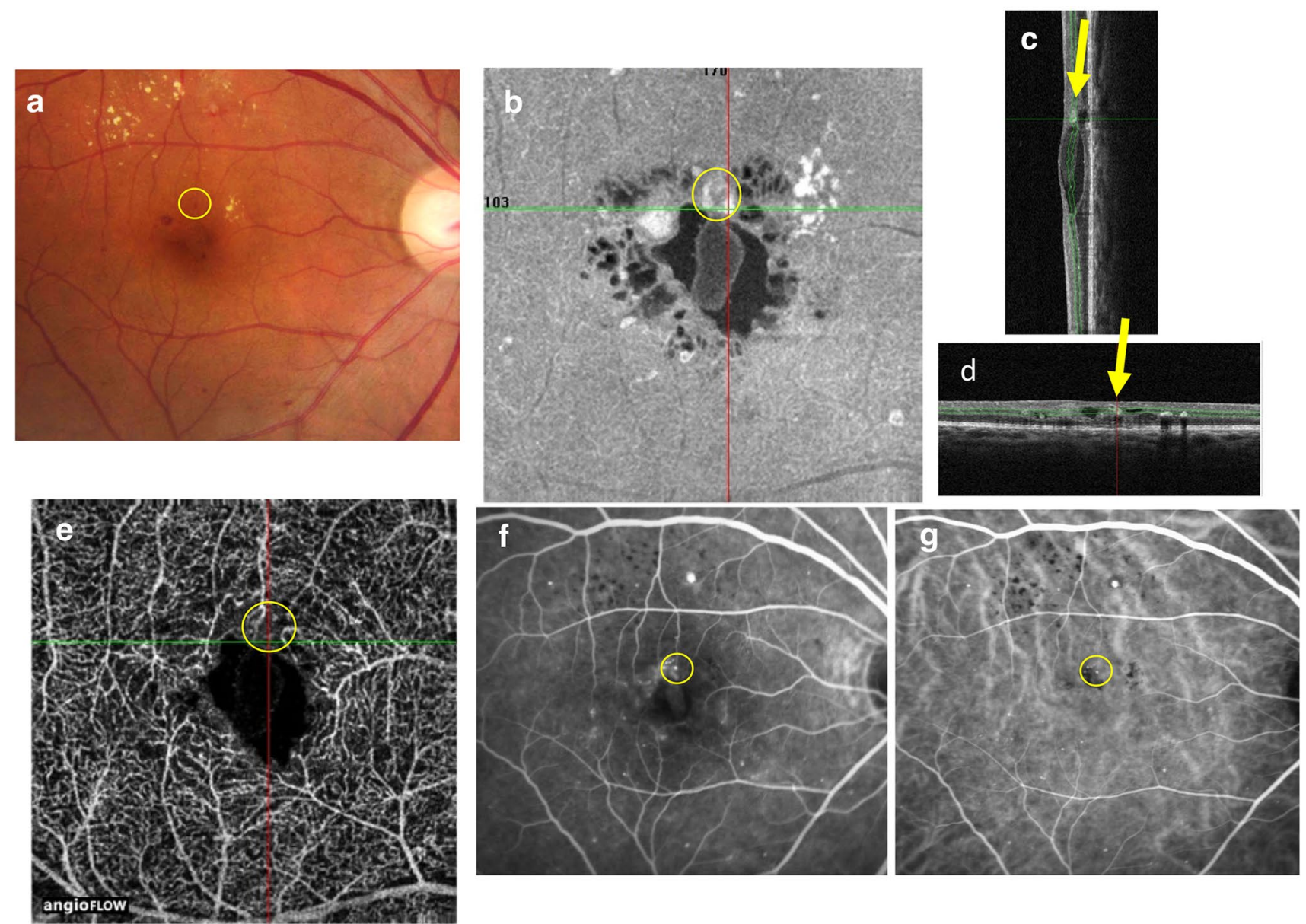

Fig. 3 A 64-year-old woman with moderate nonproliferative diabetic retinopathy of the left eye. a A fundus photograph shows a red punctate spot thought to be a microaneurysm (MA; yellow circle). b The $\mathrm{MA}$ is seen in a $3 \times 3$-mm square of an en face image (yellow circle). The MA was confirmed in optical coherence tomography (OCT) B-scan images with the $\mathbf{c}$ vertical and $\mathbf{d}$ horizontal sliders aligned. A ring-shape, thought to be the MA, can be seen. The MA lumen was open and cystoid macular edema was observed in the vicinity. e An aneurysm could not be confirmed in a $3 \times 3$-mm square OCT angiography (OCTA) en face image (deep plexus). This was defined as an absent-type MA. f The yellow circle shows hyperfluorescence in a fluorescein angiographic (FA) image (38 seconds) matching the OCTA en face image. $\mathbf{g}$ The yellow circle shows hyperfluorescence in an indocyanine green angiographic (IA) image (21 seconds) matching the OCTA en face image 